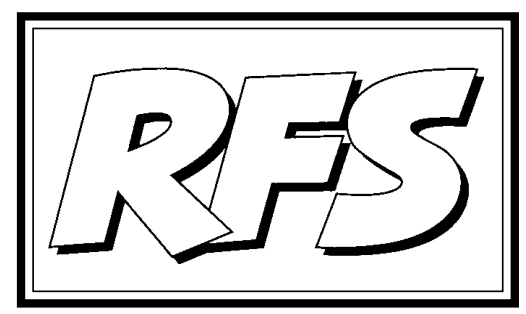

Revista de Fomento Social, 58 (2003), 191-211

\title{
Los retos de la integración regional en Centroamérica
}

Óscar Alfredo SANTAMARÍA *

El pasado 31 de marzo de 2003 se presentó en la Casa de América de Madrid la Fundación ETEA para el Desarrollo y la Cooperación con la colaboración de la Agencia Española de Cooperación Internacional (AECI). Esta nueva Fundación retoma el trabajo que ETEA (institución universitaria de la Compañía de Jesús y Facultad de Ciencias Empresariales de la Universidad de Córdoba como centro adscrito) viene realizando desde hace ya más de quince años en varios países del Sur. Las actividades de cooperación universitaria, formación de capital humano, desarrollo rural, empresa y desarrollo y políticas de desarrollo en Nicaragua, El Salvador, Guatemala, Cuba, Vietnam y otros países, han animado a ETEA a crear esta nueva estructura desdela que desarrollar más y mejores acciones en coordinación con las universidades de la Compañía de Jesús en América Latina y otros socios en los países del Sur.

* Secretario General del Sistema de la Integración Centroamericana-SICA y ex-Canciller del gobierno de El Salvador. Intervención en el evento: Centroamérica: Los Retos del Desarrollo y la Integración Regional, celebrado en la Casa de América, Madrid, España, 31 de marzo de 2003. 
De la Fundación ETEA para el Desarrollo y la Cooperación dependerá también el Instituto Español de Estudios Centroamericanos (IDECA) que coordinará las acciones en Centroamericana, región en la que ETEA ha trabajado de manera especialmente intensa en las décadas pasadas. Precisamente las jornadas se desarrollaron en torno al desarrollo de la región y su proceso de integración centroamericana.

En la J ornada participaron destacados ponentes centroamericanos como J aime Morales (Director de la Cátedra de Teoría del Estado de la Universidad de Costa Rica y Director del Centro Estudios para el Futuro-Centroamerica) y Hugo Beteta (Vicerrector Administrativo de la Universidad Rafael Landívar de Guatemala) y fue presentado el Plan regional de cooperación en Centroamérica de la $\mathrm{AECl}$ con la presencia en el acto del Director General de Iberoamérica de la AECl, Juan López-Dóriga, y Subdirector General para México, América central y caribe, Rafael Garranzo.

La ponencia del Secretario general del Sistema de la Integración Centroamérica, Oscar Santamaría, puso de relieve los avances del proceso de integración en los últimos años tras la catástrofe del Mitch y de los terremotos de El Salvador. La integración regional avanza significativamente en estos últimos años y discute ahora el establecimiento definitivo de la unión aduanera y la definición de los acuerdos de libre comercio con EEUU y de asociación con la UE. Pero los progresos del SICA van más allá de los acuerdos comerciales y de cooperación y hay algunos avances importantes en la dimensiones políticas, sociales, medioambientales y de seguridad regional. El Secretario General del SICA abordó también el reto del fortalecimiento de las instituciones regionales del proceso.

\section{Introducción}

Me es grato presentarme ante ustedes en esta jornada, con la satisfacción de anunciarles que la integración centroamericana vive uno de sus momentos más prometedores.

Tras el impasse en el proceso posterior a 1995 y mientras la región se enfrentaba a la magnitud de los daños producidos después por el Huracán Mitch y los terremotos de El Salvador, se fueron produciendo una serie de acontecimientos que han generado un amplio y rico debate en nuestros países centroamericanos. 
Debo destacar de entre todos ellos las reuniones de los Grupos Consultivos celebradas en Estocolmo en 1999 y en Madrid en el año 2001. Estas dos reuniones pusieron en evidencia la importancia y valor de sumar los esfuerzos de los gobiernos de Centroamérica, de sus sociedades civiles y de la cooperación internacional. Del Grupo Consultivo de Madrid 2001 surgió una Estrategia de Transformación y Modernización del área y un catálogo de proyectos, elaborados por todos los agentes de la región, que demostró también el valor añadido que aporta la visión regional a la solución de nuestros problemas.

Estudios de gran magnitud como el conocido como Centroamérica 2020, copatrocinado por la Unión Europea, habían creado también un ambiente de reflexión regional sobre los obstáculos que encuentra Centroamérica en su desarrollo. Esta Secretaría General (SG) impulsa hoy, con el apoyo de organismos internacionales, otros estudios de gran alcance que pueden dar un renovado impulso al proceso. Retomando aquella conocida experiencia europea de los Costos de la No Integración y con la inestimable colaboración de D. Paolo Cecchini, la SG-SICA y la CEPAL impulsan desde hace meses un estudio sobre los beneficios y costes del proceso.

Pero por otra parte, se producen en estos momentos avances muy significativos en el proceso. En los últimos meses han avanzado de forma muy notable las negociaciones para el establecimiento de la Unión Aduanera. Todo indica que a breve plazo podremos asistir al establecimiento definitivo dela Unión Aduanera Centroamericana. La Unión Europea no es ajena a estos esfuerzos y ha puesto en marcha un programa de apoyo a la Unión Aduanera que supone una importante colaboración en este trascendental paso del proceso centroamericano de integración regional.

El balance de estos años en términos de la relación económica entre los países de la región ha sido francamente positivo. Pese a un entorno complejo y cambiante, la integración centroamericana ha permitido, por ejemplo, un desarrollo desconocido del comercio intrarregional que ya superó las cifras de los 3000 millones US\$ de exportaciones entre los socios regionales.

Esta mayor interrelación entre las economías de la región es también palpable en lo que se conoce en la región como la integración informal. Algunos sectores empresariales centroamericanos avanzan en la integración de sus negocios y actividades más rápido que las instituciones. Son buenos ejemplos de ello, el sistema financiero, el sector energético, el sector agroalimentario, el sector transporte, el turístico y la distribución comercial, entre 
muchos otros. En estos sectores ya se da por hecho que la integración no es sólo necesaria sino inevitable y trabajan en función de este escenario. Es un indicador de la fortaleza creciente de la integración regional y, al mismo tiempo, un estimulo a proseguir en el desarrollo de los acuerdos.

Las negociaciones para el establecimiento de la Unión Aduanera crearán un entorno favorecedor para un mayor desarrollo del comercio intrarregional. Cabe destacar como ejemplo de ello la plena incorporación de Costa Rica a estas negociaciones y el avance notable en la ejecución del Plan de Acción económico de marzo de 2002 que comprende el calendario definitivo para alcanzar el 1 de enero de 2004 la liberalización del comercio intrarregional, la definición de un arancel común centroamericano y la eliminación de los puestos fronterizos, cumpliendo así con uno de los objetivos primordiales del Protocolo de Guatemala al Tratado General de Integración Económica Centroamericana.

Por otro lado, la red de acuerdos comerciales con terceros países y regiones se va completando progresivamente. Tras los acuerdos con México, República Dominicana y Chile; la región avanza en el establecimiento de acuerdos conjuntos con Canadá, tiene fechas concretas para finalizar un Tratado de Libre Comercio (TLC) con Estados Unidos y está a las expectativas de abrir negociaciones para un nuevo y más amplio Acuerdo de Asociación con la Unión Europea que incluiría también una Acuerdo de Libre Comercio.

Pero junto a los avances en el definitivo establecimiento de la unión aduanera, se han producido otros avances de gran importancia en el conjunto de las dimensiones de la nueva integración centroamericana. Haremos enseguida una breve reseña de los más destacados, pero quiero recuperar ahora algunas ideas esenciales sobre la integración centroamericana de nuestros días.

\section{Lecciones}

1.1. El marco institucional de la integración de los noventa: el Protocolo de Tegucigalpa

Como ustedes ya conocen, los procesos de Contadora y Esquipulas y muy especialmente el Diálogo de Cooperación de "San J osé" entre Centroamérica 
y la Unión Europea instituido en 1984, permitieron a los países centroamericanos superar los conflictos que habían afectado la experiencia de integración regional de los años sesenta y setenta, iniciada con la carta de la ODECA en 1951, cuando también la Unión Europea daba sus primeros pasos tras la creación de la Comunidad Europea del Carbón y del Acero en ese mismo año.

Las Cumbres presidenciales de los primeros cinco años de la década de los noventa, dieron lugar al nuevo marco político y jurídico de la integración regional que está presidido por el Protocolo de Tegucigalpa de 1991 por el que se crea el marco político en el que se enmarcan el resto de nuestros tratados fundamentales y se desarrolla el marco institucional: el Protocolo de Guatemala de 1993, el Tratado de la Integración Social de 1995 y el Tratado Marco de Seguridad Democrática de 1995. Este conjunto de acuerdos permitió a los centroamericanos dotarse de un conjunto de principios, normas y objetivos, centrados en la paz, el desarrollo, la libertad y la democracia.

La nueva integración de los años noventa pretendió responder a los retos del desarrollo centroamericano fomentando un escenario regional de crecimiento y de modernización de nuestras sociedades, que al mismo tiempo mejorase las condiciones de nuestra inserción en la economía mundial y en las instituciones y acuerdos que la rigen. La región optó por un modelo de crecimiento económico dual, hacia adentro con el objetivo final de lograr la Unión Económica y hacia afuera con políticas regionales que favorecen el desarrollo de las exportaciones a terceros países o grupos de países, a través de TLC's.

El conjunto de instituciones del proceso está llamado ahora a impulsar el desarrollo de las distintas dimensiones (política, económica, social, medioambiental y cultural) del proceso, mediante el pronto establecimiento de una Unión Aduanera que progresaría hacia el mercado único y el desarrollo de las políticas y acciones comunes en todos los ámbitos del proceso. Las principales novedades institucionales que generó el Protocolo de Tegucigalpa con respecto a la integración original fueron la creación de la Secretaría General, de la Corte Centroamericana de Justicia y del Comité Consultivo de la Integración Centroamericana.

En el esquema institucional centroamericano la toma de decisiones se concentra en el órgano de máximo rango que es la Reunión de Presidentes. La Corte Centroamericana de J usticia debe cumplir la función de asegurar el cumplimiento de los acuerdos centroamericanos y de ordenar el marco jurídico del proceso así como los conflictos que surjan en torno a él. 
El Parlamento Centroamericano fue un elemento esencial en la recuperación del proceso en los años ochenta. En la valoración de sus funciones actuales, se olvida con frecuencia su importancia como foro de diálogo tras su creación en una región en pleno conflicto en 1987 (años antes de que el protocolo de Tegucigalpa fuese aprobado).

La Reunión de Vicepresidentes y los Consejos de Ministros son los siguientes órganos de la institucionalidad y abarcan un amplio rango de sectores y políticas en la región. Es preciso destacar el rol del Consejo de Ministros de Relaciones Exteriores como cabeza rectora del proceso y el notable protagonismo del Consejo de Ministros de Economía.

El Comité Consultivo es otra de las novedades del marco institucional. Ha funcionado durante estos años con la presencia de numerosos agentes de la región. Su rol en el proceso es de la máxima importancia aunque sea de carácter consultivo. En la actualidad, tratamos de impulsar desde la SG-SICA su participación en los debates relacionados con la agenda presidencial y reforzar su capacidad de opinión. La conexión de la Sociedad Civil con la agenda regional y su percepción sobre el proceso es un factor fundamental para su avance.

Junto a estos órganos, la Secretaría General del SICA encabeza la red de Secretarías, Direcciones e instituciones del sistema. La coordinación de estas secretarías e instituciones se convierte en un elemento determinante para el éxito de los esfuerzos de integración.

En el diseño de este marco institucional existe, como en los propios fundamentos del modelo de integración, una enorme influencia del modelo europeo. Es por ello que las experiencias de la Unión Europea constituyen para nosotros una permanente referencia en los debates tanto sobre la reforma institucional como sobre cualquier otro aspecto del proceso. La fructífera relación entre la integración centroamericana y la Unión Europea a través del Diálogo Político y de Cooperación denominado "Foro de San José", es sin duda uno de las fortalezas de nuestro proceso. La colaboración europea se ha manifestado permanentemente desde su apoyo a los acuerdos de paz o a la creación de Parlamento Centroamericano hasta su apoyo al establecimiento de la Unión Aduanera en estos días. No puedo dejar de manifestar en esta ocasión nuestro agradecimiento por este acompañamiento. 
1.2. Aspectos positivos y negativos del marco institucional creado por el Protocolo de Tegucigalpa

Regresando al marco institucional creado por los nuevos Tratados, podríamos sintetizar sus aspectos positivos y negativos de la forma siguiente.

En cuanto a los aspectos positivos se puede afirmar:

- Que los acuerdos y tratados del Sistema de la Integración Centroamericana están basados en la recuperación de la paz regional y tienen como objeto fundamental la consolidación de un espacio de entendimiento regional que impida conflictos futuros. Como señala el Protocolo de Tegucigalpa, el objetivo fundamental es "la realización de la integración de Centroamérica, para constituirla como Región de Paz, Libertad, Democracia y Desarrollo".

- Que los acuerdos y tratados del Sistema de la Integración Centroamericana resuelven la unidimensionalidad de la etapa anterior del proceso y consagran un modelo de integración con las siguientes dimensiones: política, económica, ambiental, social y cultural.

- Que los nuevos acuerdos de integración regional en Centroamérica constituyen un marco adecuado y con aportaciones valiosas para el desarrollo regional. Por ejemplo, incorporan algunos de los principales consensos sobre desarrollo gestados durante los años noventa en la comunidad internacional. Conceptos como desarrollo sostenible, integración con apertura, desarrollo humano o seguridad humana forman parte sustancial de los tratados y acuerdos centroamericanos.

- Que la presencia de acuerdos y tratados en las dimensiones sociales y medioambientales del proceso constituye una adecuada respuesta a las necesidades de los países de la región. El Tratado de la Integración Social sitúa el desarrollo humano en el eje de la integración. La Alianza para el Desarrollo Sostenible (ALIDES) sitúa el principio del desarrollo sostenible en el eje de la integración.

- Que los nuevos acuerdos resolvieron también dos carencias importantes de la integración centroamericana con la creación de la Corte Centroamericana de Justicia, el PARLACEN y el Comité Consultivo de la Integración Centroamericana. Su presencia incorpora un valor añadido de enorme consideración al entramado institucional.

En cuanto a los aspectos negativos se puede afirmar: 
- Que existe una cierta confusión en el marco jurídico y político del proceso ante la dificultad de trabajar en un marco en que los acuerdos no son suscritos y ratificados por todos los estados miembros del SICA y en el que se constata la ausencia de mecanismos para normar la ratificación de los acuerdos.

- Que se producen en ocasiones decisiones unilaterales contrarios a los acuerdos centroamericanos así como incumplimientos de los acuerdos aprobados.

- Que la institucionalidad regional precisa efectivamente de una reforma que resuelva problemas como la dispersión institucional y su necesaria racionalización y la revisión de competencias y procedimientos de algunas de sus instituciones para reforzar su funcionamiento y capacidad.

- Quela dispersión institucional supone una pérdida de eficacia y eficiencia del entramado institucional que se debe corregir con mecanismos adecuados de coordinación entre todas las instituciones.

- Que la institucionalidad no cuenta con fuente y mecanismo de financiamiento automático.

En general, se puede afirmar que la integración centroamericana se sitúa a medio camino entre lo intergubernamental y lo comunitario. Esta característica del proceso es especialmente visible en el ámbito institucional del proceso y determinará los resultados de las reformas a realizar.

1.3. La reforma del marco institucional: la propuesta BID-CEPAL y las Cumbres presidenciales de Panamá y de Comalapa

La necesidad de corregir los aspectos negativos del proceso, animaron a los gobiernos regionales a plantear ciertas medidas de reforma institucional. La CEPAL y el BID coordinaron el Programa de apoyo al fortalecimiento y racionalización de la institucionalidad de la integración centroamericana, siguiendo un mandato de la XVI Cumbre de Presidentes celebrada el 30 de marzo de 1995 en San Salvador. Los resultados de este programa dieron lugar a una serie de propuestas sobre la racionalización y refuerzo de las instituciones comunitarias que fueron poco después discutidas y adoptadas en la XIX Reunión de Presidentes que tuvo lugar el 11 y 12 de julio de 1997 en Panamá. Los Presidentes decidieron abordar la reforma institucional del 
SICA con la racionalización como objetivo principal. Sus principales decisiones fueron las siguientes:

a) Revisar las atribuciones y el número de diputados del PARLACEN.

b) Derogar la competencia de intervención interna de la Corte Centroamericana de Justicia y reducir los magistrados a uno por país.

c) Unificación de las Secretarías del Sistema.

d) Sustitución del Comité Ejecutivo por el Comité de Enlace.

Por otro lado, los gobiernos centroamericanos decidieron iniciar los trabajos para la refundición de los acuerdos regionales en un Tratado único.

El 4 de febrero de 1998 los Presidentes centroamericanos realizaron una reunión extraordinaria en el aeropuerto de Comalapa (San Salvador) para discutir especialmente los avances en el proceso de reflexión y reforma del marco institucional. De esta cumbre surgieron al gunas decisiones importantes. Las principales fueron las siguientes:

a) Reducir el número de parlamentarios del PARLACEN de los 20 actuales a 10 o 15 por país.

b) Reducir el número de magistrados de la Corte Centroamericana de Justicia de 2 a 1 por país y modificar su relación laboral con la Corte, pasando a retribuir por dietas o sesiones y no por sueldos mensuales.

c) Proceder a la unificación de las instituciones regionales en una secretaría unificada con sede en San Salvador bajo la coordinación del Secretario General del SICA.

d) Nombrar a un técnico como Presidente del Banco Centroamericano de Integración Económica, eliminando el hábito de rotar la presidencia entre los distintos países; y modificar los estatutos del Banco para facilitar el ingreso de socios extrarregionales.

Las reformas son de gran interés desde el punto de vista de la racionalización del sistema pero generaron una importante discusión en el entorno institucional. 
1.4. Los avances en la reforma de las instituciones desde 1997 hasta la fecha: el debate sobre las reformas

Algunas de sus propuestas son efectivamente discutibles y quizás el enfoque del proyecto se ve afectado por una percepción negativa de las instituciones regionales. En cualquier caso, los documentos y decisiones presidenciales incluyen propuestas de gran valor para el proceso que merecen una segunda oportunidad.

Las decisiones presidenciales de reforma sólo han sido parcialmente abordadas. Solamente respecto a la unificación de las secretarías en San Salvador se han producido algunos avances. La Secretaría Ejecutiva de la Comisión Centroamericana de Ambiente y Desarrollo, la OSPESCA, la Secretaría de la Integración Turística y la Secretaría de la Integración Social se han desplazado a El Salvador.

Las reformas sobre el Parlamento Centroamericano y la Corte Centroamericana de Justicia no se han puesto en marcha. Tanto el Parlamento como la Corte han argumentado intensamente contra las reformas propuestas y no se han tomado decisiones firmes. Algunos gobiernos proponían recientemente la suspensión del mecanismo de elección por sufragio universal para determinar los parlamentarios por designación de entre los parlamentarios nacionales.

Pese a la conveniencia de valorar las decisiones presidenciales, es importante señalar que con frecuencia se exige a las instituciones centroamericanas resultados en poco tiempo y sin muchas competencias para alcanzarlos. Una vez más el caso europeo nos debe servir de inspiración para dar tiempo a la integración y a sus instituciones para mostrar su eficacia en el medio plazo.

Más allá de las propuestas, es preciso revisar para mejorar y reforzar el rol en el proceso de instituciones como el PARLACEN, la Corte Centroamericana de Justicia o el Comité Consultivo.

Esta Secretaría General la mantiene una mesa de coordinación interinstitucional con PARLACEN y la Corte Centroamericana con el interés de facilitar su plena participación en la agenda centroamericana y de coordinar la labor de las tres instituciones.

Recientemente se ha producido una reforma de los protocolos de Tegucigalpa y de Guatemala para convertir al Consejo de Ministros responsables de 
la integración económica en instancia de solución de las controversias comerciales, instancia que también incorpora un mecanismo de arbitraje.

Conviene asimismo destacar que la Secretaría General del SICA con el apoyo técnico de expertos de la Unión Europea, realiza desde el año 2002, un proceso de reestructuración y modernización organizacional, con el objeto de fortalecerla y actualizarla orgánica y operacionalmente, estableciendo sistemas de planificación estratégica, informática, control, seguimiento, evaluación y retroalimentación de sus actividades, proyectos y programas, que facilitaran la programación del proceso en forma dinámica en el corto y medio plazos.

1.5. El falso mito de la institucionalidad ineficiente: algunos casos positivos que demuestran la capacidad de las instituciones regionales

Las instituciones centroamericanas están sujetas a veces a un cierto mito negativo que conviene contrarrestar. Existen múltiples ejemplos de los avances que se producen en el proceso y es fácil establecer su relación causal con instituciones que los apoyan con capacidad de propuesta y de organización. Es decir, hay tantos o más ejemplos positivos que negativos que demuestran la eficacia del sistema.

Más allá de los avances ya reseñados, se han producido otros progresos con protagonismo institucional destacado. Por ejemplo, podemos citar los siguientes:

En lo que respecta al ámbito económico conviene destacar especialmente el "Plan de Acción Económico" aprobado por una Cumbre de Presidentes del área el año pasado que se presenta a continuación:

1. NORMATIVA

- SOLUCIONES DE CONTROVERSIAS COMERCIALES

- ÉNVIO AL LEGISLATIVO

- VIGENCIA

INMEDIATO

$31 / 12 / 02$

- COMERCIO DE SERVICIOS E INVERSIONES

- SUSCRIPCIÓN Y ENVÍO AL LEGISLATIVO DEL TRATADO

CENTROAMERICANO INMEDIATO

- VIGENCIA

$31 / 12 / 02$ 
- CODIGO ADUANERO UNIFORME CENTROAMERICANO (CAUCA)

- VIGENCIA

- CONCLUIR ADOPCION REGIONAL DEL RECAUCA

2. ARMONIZACION ARANCELARIA

- RUBROS CON DIFERENCIA DE 5\% O MENOS

- RESTO DE RUBROS ARANCELARIOS

- ARANCELES DIFERENTES EN NICARAGUA POR LEY DE JUSTICIA TRIBUTARIA

3. LIBRE COMERCIO PARA TODOS LOS PRODUCTOS PENDIENTES

- HARINA DE TRIGO

- CAFÉ TOSTADO

- DERIVADOS DEL PETROLEO

- ALCOHOL ETÍLICO

- BEBIDAS ALCOHOLICAS DESTILADAS

4. NEGOCIACIONES COMERCIALES

- EN PROCESO

- CANADA

- ESTADOS UNIDOS

- ALCA

- OMC

- A CORTO PLAZO

- UNION EUROPEA

- CARICOM

- MERCO-SUR

- COMUNIDAD ANDINA

5. FACILITACION DE COMERCIO

- ELIMINACION DE OBSTACULOS AL COMERCIO

- MODERNIZACION DE LAS ADUANAS Y SIMPLIFICACIÓN DE TRAMITES EN LOS PUESTOS FRONTERIZOS

6. UNION ADUANERA

- RECONOCIMIENTO MUTUO DE LOS REGISTROS

SANITARIOS DE MEDICAMENTOS

$31 / 12 / 02$

- POLITICAS AGROPECUARIAS

- ADMINISTRACION ADUANERA COMUN 
- LIBRE MOVILIDAD DE BIENES SIN EXCEPCION Y

$31 / 12 / 03$ SERVICIOS ASOCIADOS AL COMERCIO DE BIENES

- POLITICA COMERCIAL EXTERNA COMUN ${ }^{1}$

- MECANISMO DE RECAUDACION, ADMINISTRACIÓN Y

$31 / 12 / 03$

DISTRIBUCIÓN DE INGRESOS TRIBUTARIOS POR COMERCIO EXTERIOR ${ }^{1}$

- ELIMINACION DE PUESTOS FRONTERIZOS INTRACENTROAMERICANOS ${ }^{1}$

1 Son requisitos indispensables el combate al contrabando y la evasión fiscal; y que los ingresos por concepto arancelario no disminuyan en cada uno de los países

- También se avanza en la convergencia de políticas monetarias y financieras, en el ámbito del Consejo Monetario Centroamericano. La Secretaría del Consejo monetario centroamericano, las superintendencias de bancos de la región y el $\mathrm{BCIE}$, han concretado avances en el análisis de la legislación vigente en materia de supervisión financiera en los países del área, con el objeto de modernizarla, coordinarla y ajustarla a los principios de Basilea. Se analiza también la arquitectura de los mercados de valores del área y el CMCA acordó ya recomendaciones específicas con la meta de conformar el "Mercado Regional de Valores" cuya aplicación empieza con el establecimiento de un mercado regional de valores de títulos de deuda pública.

- Los avances en el sector turístico muestra un gran dinamismo. Se ha adoptado el sistema centroamericano de certificación para la sostenibilidad turística y se aprobó el plan de acción para su ejecución.

En una reciente reunión del Consejo de Ministros de Turismo, los países centroamericanos decidieron el inicio de operaciones de la nueva Agencia de Promoción Turística de Centroamérica (CATA) con sede en Madrid y con la función de promover conjuntamente Centroamérica como destino turístico y para desarrollar acciones bajo la nueva marca común "Centroamérica, tan pequeña, ... tan grande...".

- Asimismo se ha dado inicio en este año 2003 al proyecto de interconexión eléctrica en Centroamérica, definido antes del grupo Consultivo de Madrid, reforzado en la estrategia regional derivada del proceso de Madrid y ahora desarrollándose en el ámbito del Plan Puebla Panamá que ha retomado algunos de los proyectos del catálogo regional. 
En lo que respecta al ámbito social, se pueden mencionar los siguientes ejemplos de buen desempeño institucional:

- El Consejo de Ministros del área social aprobó la propuesta de "lineamientos de políticas y estrategias para el desarrollo y la integración social" para el período 2000-2020, estableciendo metas para el primer decenio. Igualmente, dando continuidad al esfuerzo del proceso de Madrid, aprobó una cartera regional de proyectos para el desarrollo social, derivada de los lineamientos anteriores. Ha servido además de base para la propuesta social en el marco del Plan Puebla Panamá.

- Asimismo consideramos de especial importancia destacar las negociaciones realizadas por el Consejo de Ministros de Salud de Centroamérica, COMISCA, que llevaron a obtener rebajas históricas en la compra de medicamentos para tratar el VIH/SIDA. Este logro tiene como resultado concreto la posibilidad de triplicar la cobertura de atención a estos pacientes en la región. Esto, se consiguió gracias al trabajo conjunto desarrollado por los Ministerios de Salud de Centroamérica y apoyados por la Secretaría Técnica de Integración Social, parte integral de la SG-SICA.

En lo que respecta al ámbito medioambiental, sirven como ejemplo los siguientes avances:

- La integración medioambiental se dotó del Ilamado Plan Ambiental de la Región Centroamericana (PARCA). De su cartera de proyectos se ha lanzado ya el proyecto "Sistema Arrecifal Mesoamericano"; el Programa Ambiental Regional para Centroamérica (PROARCA) que busca un manejo ambiental mejorado del Corredor Biológico Mesoamericano; así como el Programa de Modernización de la Gestión Ambiental en Centroamérica (PROSIGA) con la armonización de marcos regulatorios ambientales en la región centroamericana.

- En cuanto a la prevención y mitigación de desastres, la $X$ reunión de Presidentes centroamericanos celebrada en Guatemala en octubre de 1999 adoptó un modelo regional para la reducción de la vulnerabilidad y los desastres en Centroamérica y decidieron establecer un quinquenio centroamericano para la reducción de las vulnerabilidades y el impacto de los desastres para el periodo 2000 a 2004.

Y finalmente, en los que respecta al ámbito político, se pueden señalar los siguientes avances: 
- En materia de seguridad, la región ha alcanzado logros concretos a través de los trabajos desarrollados por la Comisión de Seguridad de Centroamérica, así como por las Subcomisiones de Seguridad, Defensa y J urídica de la misma. En este contexto, se esta desarrollando un modelo de seguridad democrática donde destacan principalmente la adopción del "mecanismo regional de coordinación de ayuda mutua ante desastres", así como el "plan regional contra el crimen organizado".

- Por otro lado, es importante destacar la elaboración del "plan centroamericano de cooperación integral para prevenir y contrarrestar el terrorismo y actividades conexas", el cual fuera adoptado en resolución dela Comisión de Seguridad de Centroamérica el 25 de octubre de 2001.

\section{Retos de la Integración Centroamericana}

2.1. La potencialidad de la institucionalidad centroamericana: ¿por qué son imprescindibles las instituciones regionales?

Quizás el mejor ejemplo de la lenta pero progresiva consolidación del SICA ha sido la capacidad demostrada por las instituciones y gobiernos regionales para elaborar la "Estrategia regional de transformación y modernización de Centroamérica para el siglo XXI" y presentarlo en la reunión del Grupo Consultivo de Madrid en marzo de 2001. El difícil entorno en el que se elaboró esta Estrategia es una manifestación de las dificultades que encuentra el proceso de integración. Pero pese a ello, la región pudo elaborar una estrategia regional para enfrentarse conjuntamente a los obstáculos del desarrollo centroamericano.

El proceso de Madrid reunió bajo la coordinación de las instituciones regionales a gobiernos, sociedad civil y cooperación internacional, para elaborar una Estrategia de Transformación y Modernización de Centroamérica y un catálogo de 31 proyectos que responden a los factores críticos para el desarrollo regional.

El proceso de Madrid puso de manifiesto la potencialidad de los mecanismos del SICA para la coordinación de gobiernos e instituciones regionales cuando estos se pueden desarrollar con toda la amplitud que contemplan los acuerdos y el propio marco de la integración regional. 
Gobiernos e instituciones trabajamos ahora en la ejecución de algunos de estos proyectos cuyo desarrollo depende de la coordinación de los esfuerzos que realicemos en la región y de los compromisos de apoyo que los países y organizaciones amigas están haciendo en torno a ellos.

Quiero insistir en el valor que esta SG-SICA concede a la "Estrategia regional de transformación y modernización de Centroamérica para el siglo XXI" como contenido central de la integración centroamericana.

Numerosos son, por tanto, los avances en el proceso de integración. Todos ellos permiten afirmar que el año 2002 ha sido un buen año para la integración regional. Los gobiernos han incrementado sus expectativas sobre la acción conjunta y las perspectivas para el año 2003 son igualmente halagüeñas por todo lo comentado anteriormente.

Por todo ello, podemos desplegar un moderado optimismo, sólo tendríamos que limitarlo por asuntos como la gravedad de la crisis del café y la situación actual del entorno internacional.

A estemoderado optimismo nos puede ayudar otro de los logros importantes, que quisiera destacar especialmente. La SG-SICA realizó, a mediados del año pasado y con la firma CID-Gallup, la primera investigación de opinión ciudadana en el ámbito centroamericano. La investigación fue importante porque nos confirmó que nuestros pueblos perciben la integración como necesaria y conveniente para nuestros países. Un $66 \%$ de los centroamericanos estima que sería conveniente que los países de la región se presenten ante el resto del mundo como un bloque de naciones hermanas y un $60 \%$, en promedio, cree que la integración beneficia mucho 0 algo a las poblaciones de la región. Los resultados de esta encuesta ayudan a determinar el verdadero sentimiento e importante arraigo que la integración regional tiene en los ciudadanos centroamericanos. Es, sin duda, un elemento esperanzador para el futuro.

Pero también los ciudadanos mostraron un conocimiento superior a lo que se podría afirmar sobre el marco institucional de la integración centroamericana y una mayor confianza en ellas de lo que se suele afirmar.

Las expectativas de los ciudadanos y la positiva evolución del proceso implican una gran responsabilidad para las instituciones de la integración que me honro en representar. 
2.2. Eliminando los obstáculos que reduce la eficacia de la institucionalidad: el tránsito a lo comunitario. El presupuesto institucional

Nuestras instituciones se ven limitadas porque el proceso de integración regional se encuentra en un escenario de tránsito entre lo intergubernamental y lo comunitario. Los tratados construyen un escenario tendente a lo comunitario, con instituciones sobre las que se deposita capacidad de decisión y ejecución. Sin embargo, la práctica de nuestra integración todavía es marcadamente intergubernamental. Por esto las instituciones, más allá de sus limitaciones, se ven obligadas a trabajar en un entorno con cierta indefinición, con objetivos de cierto alcance contemplados en los tratados pero con competencias limitadas por la intergubernamentabilidad de los procesos.

La Integración Centroamericana y sus instituciones necesitan situarse en una etapa de tránsito a lo comunitario e ir ganando competencias y claridad de funciones. Los gobiernos de la región en este escenario de tránsito deberían ir incrementando su confianza en las instituciones, en su capacidad de definir propuestas de efecto positivo para el interés general y de ejecutarlas adecuadamente.

Para ello es preciso corregir un factor que limita continuamente la capacidad de las instituciones y que es el presupuestario. Por una parte, es preciso establecer mecanismos automáticos de financiación para los presupuestos institucionales que reduzcan la dependencia de decisiones discrecionales. Por otra parte, es preciso dotar a las instituciones de medios para que cumplan sus funciones. También en este aspecto se hace necesario optar por instituciones con los recursos económicos y humanos suficientes.

Una vez alcanzado el establecimiento de la Unión Aduanera, es preciso determinar la posibilidad de que los presupuestos institucionales provengan de la renta aduanera generada por el arancel externo común. El caso europeo es de nuevo una valiosa referencia.

2.3. La Presidencia Pro-Témpore como elemento esencial para la conducción de la agenda de la integración. La Troika

Precisamente la coordinación es un principio básico al plantearnos los desafíos de la reforma institucional. Como ustedes saben, las Cumbres Presidenciales marcan de manera muy determinante la agenda centroameri- 
cana. Por ello, su seguimiento se convierte en un mecanismo fundamental para el adecuado progreso de los acuerdos regionales. La Presidencia Protémpore viene siendo asumida por los países centroamericanos con un creciente interés que demuestra la mayor confianza y dedicación a la integración.

El impulso que el país que preside puede dar a la agenda presidencial durante su semestre está convirtiéndose en un elemento muy positivo para el proceso. La reciente presidencia de Costa Rica, la actual de Panamá y el interés con el que Belice prepara la siguiente, son muestras de la importancia de este mecanismo.

Los gobiernos centroamericanos estudian ahora la propuesta que realizamos en la XXI Cumbre de Granada (Nicaragua), sobre la conveniencia de establecer un sistema de Troika en Centroamérica, que involucre al Estado que ha ejercido la Presidencia Pro-témpore en el período anterior, al país que la ejerce actualmente y al país que tendrá en sus manos la próxima presidencia.

Como ustedes en la UE ya han experimentado sobradamente, ello permitiría que el país que asume funciones se beneficie de la experiencia de la presidencia anterior, y a su vez, la futura presidencia siga el hilo del proceso $y$, por consiguiente, asuma plenamente enterada de las acciones realizadas y los asuntos pendientes. Luego de una primera ronda, este esquema podría sustituirse por una troika donde la anterior presidencia puede ser sustituida por la Secretaría General, quedando únicamente la presidencia actual y la entrante, junto al órgano de la secretaría general.

Mecanismos como la Troika y la Presidencia Pro-témpore son ahora esenciales para garantizar un seguimiento fluido de la agenda presidencial.

2.4. El rol de la Secretaría General del SICA como coordinadora e impulsora de la agenda centroamericana y su seguimiento

La propuesta de una SG unificada en la reforma se basaba en que la dispersión de las instituciones resta una parte de la capacidad técnica y política que la Secretaría podría tener. Las distintas secretarías especializadas del sistema han estado funcionando con una difícil coordinación y sin unir los escasos recursos de que disponen. La figura de una Secretaría General del Sistema, rodeada de un equipo reducido de secretariados 
especializados, con Representaciones en cada capital de los países miembros, y funcionando con objetivos comunes, permite pensar en una institución regional con peso para garantizar el cumplimiento y progreso de los acuerdos, junto a la Corte y el Parlamento Centroamericano.

Es igualmente importante disponer de una institucionalidad coordinada desde la SG-SICA que pueda responder en la preparación y seguimiento de las decisiones de las reuniones Cumbres y de Consejos Ministeriales. El modelo de integración que definen los tratados de la nueva integración no es factible en un marco institucional como el actual. La multidimensionalidad que confieren al proceso sus ámbitos político, económico, social y cultural carga de contenido a las instituciones. La Secretaría General del SICA y la demás secretarías técnicas y especializadas no ejercen solamente la función de secretaría intergubernamental de los Consejos de Ministros, son ya en la práctica, el germen de instituciones comunitarias con un mayor nivel de capacidad de gestión y una notable capacidad de propuesta.

El marco institucional centroamericano tiene un importante papel que desarrollar en este proceso. La completa definición del organigrama institucional y la dotación de los recursos necesarios es un factor de fortalecimiento de la integración. La posición central de la SG-SICA como impulsor de iniciativas y propuestas a los gobiernos centroamericanos y como eje del seguimiento de la Agenda Presidencial y las agendas sectoriales, sería una garantía de eficacia institucional.

\section{Presencia de España en el Proceso de Integración Centroamericana}

Las relaciones comerciales, políticas y culturales, entre los países de Centroamérica y España han sido siempre muy estrechas y con las crisis políticas y económicas que los países centroamericanos atravesaron en las décadas de los años 1980 y 90, España desde esa época inició un proceso de cooperación bilateral con estos.

Ya superados los problemas de esas dos décadas, principalmente los conflictos internos en Nicaragua, El Salvador y Guatemala, el proceso de integración de los países centroamericanos cobró un nuevo impulso como herramienta insustituible de su desarrollo. En ese orden de ideas España decidió el presente año ofrecer a la región un "Programa de Cooperación Regional con Centroamérica", que se detalla a continuación: 


\subsection{Programa de Cooperación Regional con Centroamérica}

Al margen de la XII Cumbre Iberoamericana realizada el 15 y 16 de noviembre de 2002, en República Dominicana, el gobierno de el Reino de España presentó a los gobernantes centroamericanos un Programa de Cooperación Regional con Centroamérica, que evitando duplicar apoyos de otras fuentes de la cooperación internacional y dentro de una priorización consultada con los gobiernos de los países del área y las instituciones principales del Sistema de la Integración Centroamericana (SICA), comprende las siguientes áreas temáticas:

- Fortalecimiento y modernización de las administraciones públicas

- Salud

- Educación

- Desarrollo sostenibley gestión medioambiental y de recursos naturales

- Prevención de desastres y reducción de la vulnerabilidad ecológica

- Turismo

- Microempresa

- Pesca

Los proyectos y actividades que se desprendan de este programa de cooperación actual comienzan a desarrollarse con trabajos de diagnóstico en cada una de las áreas especificadas. Todos estos son sectores de gran prioridad para el desarrollo integrado de la región.

3.2. Cooperación entre la Agencia Española de Cooperación Internacional ( $\mathrm{AECl}$ ) y la Secretaría General del Sistema de la Integración Centroamericana

El 9 de marzo de 2001 se suscribió un Memorándum de Entendimiento entre la $\mathrm{AECl}$ y la SG-SICA que era el marco general de la cooperación que AECl otorgará a la SG-SICA.

El próximo 1 de abril se celebrará en esta ciudad la I Reunión de la Comisión Mixta AECI/ SG-SICA al amparo de aquel Memorándum.

En principio se han identificado tres áreas prioritarias de cooperación:

- Difusión y promoción de la integración; imagen corporativa. 
- Creación de una Biblioteca - Centro de documentación en la sede de la Secretaría General del Sistema de la Integración Centroamericana.

- Programa de formación y capacitación en integración.

De esta manera se está cubriendo un vacío muy grande en la región, cual es el del conocimiento a profundidad y la divulgación a niveles populares de la marcha del proceso integracionista así como el fortalecimiento institucional de la Secretaría General del SICA.

\section{Conclusión}

Así avanza el Proceso de Integración de Centroamérica, que se inició en los años 1960 con cinco países: Costa Rica, El Salvador, Guatemala, Honduras y Nicaragua y ahora con la adición de Panamá y Belice como Miembros Plenos y de la República Dominicana como observadora y en breve plazo incorporada con carácter de Estado Asociado en igualdad con el resto de los países.

Podemos concluir que el Proceso de la Integración Centroamericana, enfrenta problemas y desafíos serios y complejos, pero no insuperables, dentro de un accionar multinacional unificado en sus intereses y objetivos. Los desafíos se han ido atendiendo y los que aún resta solucionar se estarán atendiendo gradual pero dinámicamente.

En estas tareas Centroamérica necesita el continuado apoyo, orientación y acompañamiento que España le brinda y que la región valora como un elemento crítico en el éxito de su proceso integrador.

Muchas gracias. 